\title{
Selection of an Automated Morphological Gradient Threshold for Image Segmentation*
}

\author{
Francisco Antonio Pujol López ${ }^{1}$, Juan Manuel García Chamizo ${ }^{1}$, \\ Mar Pujol López ${ }^{2}$, Ramón Riza Aldeguer ${ }^{2}$, and M.J. Pujol ${ }^{3}$ \\ 1 Depto. Tecnología Informática y Computación \\ Universidad de Alicante, Ap. Correos 99, 03080 Alicante, España \\ \{fpujol, juanma\}@dtic.ua.es \\ 2 Depto. Ciencia de la Computación e Inteligencia Artificial \\ Universidad de Alicante, Ap. Correos 99, 03080 Alicante, España \\ \{mar,rizo\}@dccia.ua.es \\ 3 Depto. Matemática Aplicada \\ Universidad de Alicante, Ap. Correos 99, 03080 Alicante, España \\ mjose@ua.es
}

\begin{abstract}
Segmentation is an essential part of practically any automated image recognition system, since it is necessary for further processing such as feature extraction or object recognition. There exist a variety of techniques for threshold selection, as it is a fast, simple and robust method. Threshold value will have considerable effects on the boundary position and overall size of the extracted objects. In this work, we propose an automated thresholding selection, which takes into account the local properties of a pixel. To do this, the algorithm calculates the morphological gradient and Laplacian and, afterwards, chooses a suitable threshold after estimating the lowest distance between the ideal segmentation and the morphological gradient thresholding segmentation.
\end{abstract}

\section{Introduction}

The recognition of objects in images is a non-trivial task. Machine learning research involves training a classifier with data obtained from known images, and then predicting the label of test samples from unseen images. The task of decomposing an image into distinctly different but homogeneous parts is called image segmentation, and the process of extracting information from these regions that define their unique properties is called feature extraction.

Image segmentation has been the subject of intensive research and a wide variety of segmentation techniques have been reported in the literature (see [1]). It is a crucial issue since it is the first step of the image understanding process, and all others steps, such as feature extraction and recognition, depend heavily on its results.

* This work has been supported by the Spanish (CICYT), project TIC2001-0245-C0202 and by the Generalitat Valenciana, projects GV04B685,GV04B634. 
In general, the segmentation algorithms are based on two important criteria: the homogeneity of the region and the discontinuity between adjacent disjoint regions. Although many image segmentation approaches deal with these problems $([2],[3])$, it is difficult to satisfy all the properties for the optimal set of segmented regions. The resulting segmented images generally depend on the predetermined threshold values. So the algorithm often fails due to it either merges regions that must be divided or separates connected regions.

Consequently, in this work, we propose a fast, robust method for image segmentation that takes into account some local properties of every pixel to calculate a well-suited threshold; thus, our segmentation scheme gives good results even when the tests are performed with noisy real-world images. The paper is organized as follows: Firstly, we define a method to obtain the morphological gradient threshold (MGT) image segmentation in Section 2. As a result, each of the steps necessary to develop this algorithm are explained in Section 3. Then, Section 4 establishes a measure related to the appropriateness of our technique. Afterwards, in Section 5 the experimentation is widely illustrated to verify the accomplishment of our research objectives. Finally, we conclude with some important remarks in Section 6.

\section{MGT Algorithm for Image Segmentation}

Designing an image segmentation scheme needs the consideration of two features of visual recognition: cost and uncertainty. Visual recognition is generally costly because the image data is large. Visual information contains uncertainty from many sources such as discretization. In general, the more observation a vision system performs, the more information is obtained, but the more cost is required. Thus, a trade-off must be considered between the cost of visual recognition and the effect of information to be obtained by recognition. In relation to this, some of the most popular approaches which provide low computation times and good information are the threshold techniques and the edge-based methods [4].

Threshold techniques, which make decisions based on local pixel information, are effective when the intensity levels of the objects are exactly outside the range of the levels in the background. These thresholding algorithms are simple and give very good results, but deciding the threshold values is not easy. Specially, this is a really serious problem for an automated vision system, as the system should decide the threshold values taking its own decision.

On the other hand, edge-based methods (e.g., gradient operators) focus on contour detection. They involve finding the edges of objects in the image and using this edge information to achieve the complete boundaries for the main objects in the image. Edge detection has many problems, especially when working with noisy images, since it could even fragment the true edges.

To overcome these problems, we propose a method that combines both thresholding and gradient operators: the so-called Morphological Gradient Threshold (MGT) segmentation, as described in Table 1. It consists of 7 main steps, where the gradient and the Laplacian are calculated in terms of Mathematical 
Morphology operations and the optimal threshold value is selected by measuring the lowest distance between the ideal segmentation and a collection of MGT segmented images.

Table 1. MGT segmentation algorithm.

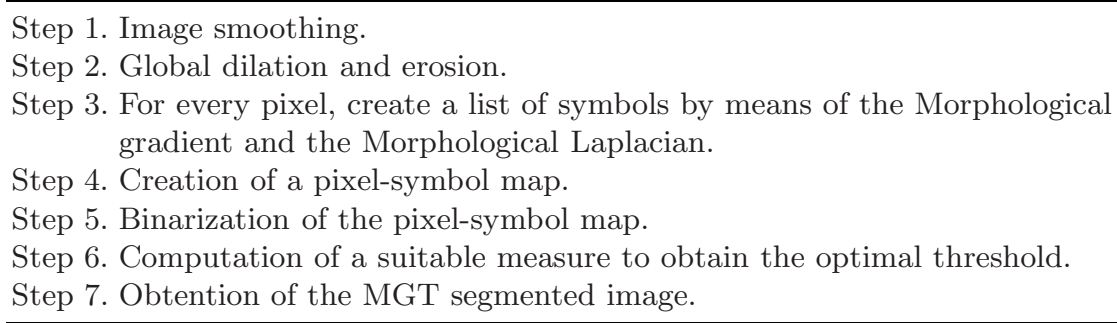

Next, a more detailed description of this process is found.

\section{Construction of a Pixel-Symbol Map}

\subsection{Image Smoothing and Derivative-Based Operations}

In every digital image there is a certain amount of white noise. To avoid the noise effects, which only consume computation time and affect the real image features, an initial filtering process has to be applied. There are many algorithms to accomplish this task; in our approach a Gaussian filter has been chosen, since it preserves many of the image features while its computational cost can be assumed in a real-time environment. For more information see [5].

Once the noise is eliminated, the point is how to create the pixel-symbol map. To do this, let us consider first the computation of some derivative-based operations, i.e., the gradient and the Laplacian.

As mentioned before, edge detection is a main problem in image analysis. There are many approaches to obtain edges by means of the gradient of an image (e.g., Prewitt or Sobel operators). Among all of these methods we find the morphological gradient, which uses the Mathematical Morphology operators.

Mathematical Morphology (MM) is a geometric approach in image processing and, originally, it was developed as a tool for shape analysis. The two elementary operations in MM are erosion and dilation [6]; from this two primitives, more complex operations are constructed, such as opening, closing and top-hat. Therefore, one can define the morphological gradient of an image $X$ by a structuring element $(\mathrm{SE}) B, \rho_{B}(X)$, as:

$$
\rho_{B}(X)=\frac{1}{2}\left(\delta_{B}(X)-\varepsilon_{B}(X)\right) .
$$

where $\delta_{B}(X)$ and $\varepsilon_{B}(X)$ are, respectively, the dilation and the erosion of an image $X$ by a SE $B$. 
It is well-known that MM has not been usually utilized when working with real-time images, as it has a very high running time. In [6] it is described an algorithm which overcome this problem by taking into account all the pixels in an image at the same time.

Let us consider that an image is dilated, using a line-segment SE that consists of two points separated by $n$ pixels. Then, we define a projection matrix, $M_{p}$, built from the original image moved $n+1$ points to the right/left, depending on the location of the SE center. Considering a binary image, one should do the logical operation OR between the original image and the moved one in order to obtain the required dilation. This method can be extended to dilations with any 2-D SE and, more generally, to the erosion, where the logical operation AND is used instead of the OR one. In gray-scale images, OR/AND operations should be replaced by the supremum/infimum ones. The computational cost of this approach is even $50 \%$ better than the classical MM method [6].

The following step is to calculate the second derivative, the Laplacian. Again, we have chosen a morphological implementation for the Laplacian, as we can use with costless time the previously pre-calculated erosion and dilation. Thus, the morphological Laplacian of an image $X$ by a $\mathrm{SE} B, \Lambda_{B}(X)$, is defined as:

$$
\Lambda_{B}(X)=\frac{1}{2}\left(\delta_{B}(X)+\varepsilon_{B}(X)\right)-X .
$$

The results for a gray-scale image after these initial steps are shown in Fig. 1, where the $\mathrm{SE} B$ is a $3 \times 3$ square.

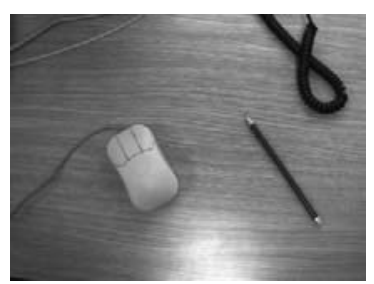

(a)

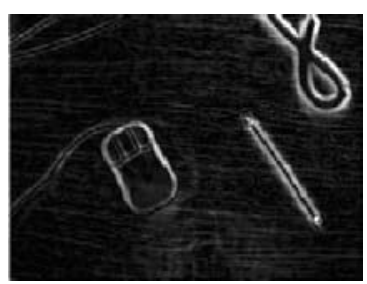

(b)

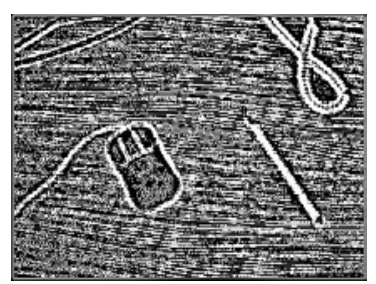

(c)

Fig. 1. A real image: (a) Original image. (b) $\rho_{B}(X)$. (c) $\Lambda_{B}(X)$.

\subsection{The Pixel-Symbol Map}

The next task is building a map that characterizes properly the pixels for a good segmentation. Thus, the pixel-symbol map $m(x, y)$ is obtained as follows:

$$
m(x, y)=\left\{\begin{array}{lll}
128 & \text { if } & \rho_{B}(x, y)<M G T \\
255 & \text { if } & \rho_{B}(x, y) \geq M G T \text { and } \Lambda_{B}(x, y) \geq 0, . \\
0 & \text { if } & \rho_{B}(x, y) \geq M G T \text { and } \Lambda_{B}(x, y)<0
\end{array}\right.
$$

where $M G T$ is the morphological gradient threshold and $(x, y)$ is a pixel in $X$. The resulting image has three different gray-levels, according to if a pixel belongs to an object, to the background or to the borders. 
The choice of the threshold value is one of the most difficult tasks, since the final result is high dependent on many factors, such as lighting conditions, objects texture or shading. Fig. 2 shows the results of the construction of the pixel-symbol map for the image in Fig. 1, with several different $M G T$ values.

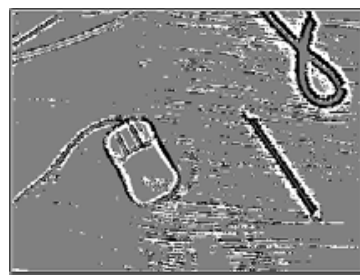

(a)

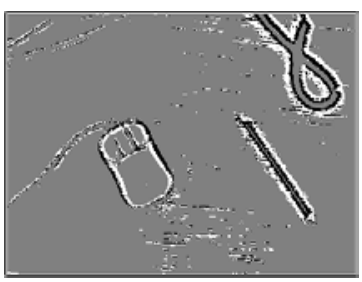

(b)

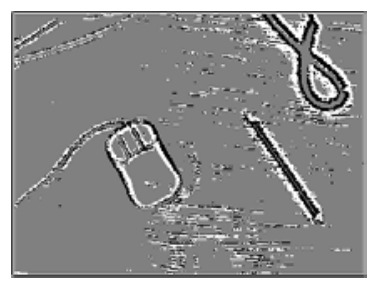

(c)

Fig. 2. The pixel-symbol map $m(x, y)$ with different $M G T$ values: (a) Gradient mean. (b) $M G T=0.9 * \max \left(\rho_{B}(x, y)\right)$. (c) $M G T=0.8 * \max \left(\rho_{B}(x, y)\right)$.

Though many practical systems utilize an experimentally obtained threshold, in this work we consider the use of an automated thresholding system. This method takes into account a binary image metrics to compare the segmentation results and, afterwards, to establish the quality level of the obtained segmentation, as it is described in the following section.

\section{A Measure of the Quality of the Segmentation}

A main problem in computer vision is to be able to compare the results using a proper metrics. This will quantify the differences between two images and, if binary images are used, the method would be both easily implementable and low computationally complex. In our system we are interested in measuring the distance between image $G$ (the map after gradient thresholding) and image $A$ (the ideal segmentation). Thus, it will establish the optimal $M G T$ value.

Hence, the map must be binarized first. To do this, we must recall that $m(x, y)$ has only 3 gray-levels (Eq. (3)): 0, 128 and 255. For simplicity, let us consider that the threshold is the same as in the construction of $m(x, y)$, i.e., the gradient threshold $M G T$. The results of this process are shown in Fig. 3.

Next, a reliable measure to compare the obtained image segmentation with an ideal segmentation must be selected.

As proved in [7], a good error measurement for binary images is $\Delta^{p}(A, G)$, defined as the $p^{\text {th }}$ order mean difference between the thresholded distance transforms of two images: $A$ (the ideal segmentation) and $G$ (the binary pixel-symbol map). Let us define first some previous terms:

- Let $X$ denote the pixel raster.

- A binary image $A \subseteq X$ is a set $A=\{x \in X: A(x)=1\}$. 


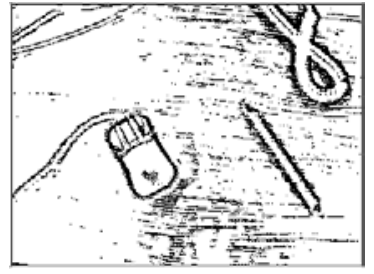

(a)

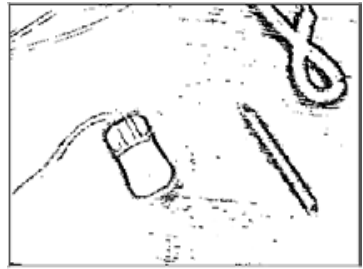

(b)

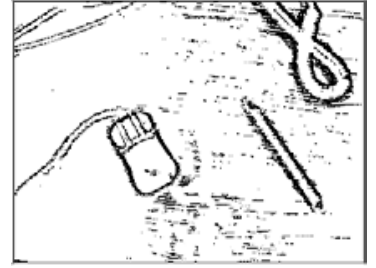

(c)

Fig. 3. Binarization with different $M G T$ values: (a) Gradient mean. (b) $M G T=0.9 *$ $\max \left(\rho_{B}(x, y)\right)$. (c) $M G T=0.8 * \max \left(\rho_{B}(x, y)\right)$.

If $\varrho(x, y)$ is the distance between two pixels $x$ and $y$, the shortest distance between a pixel $x \in X$ and $A \subseteq X$ is defined as:

$$
d(x, A)=\inf \{\varrho(x, a): a \in A\} .
$$

Then, for $1 \leq \varrho \leq \infty$ we define:

$$
\Delta^{p}(A, G)=\left[\frac{1}{N} \sum_{x \in X}|w(d(x, A))-w(d(x, G))|^{p}\right]^{1 / p} .
$$

where $N$ is the total number of pixels in $X$ and $w(t)=\min (t, c)$, for $c>0$.

Intuitively, $\Delta^{p}(A, G)$ measures the suitability of an estimated image to be used instead of the real one.

Now, we can evaluate the goodness of our segmentation scheme.

\section{Experiments}

Let us show now the results of some experiments completed for our model. The tests have been performed with a set of real images, whose pixel-symbol maps have been calculated for different $M G T$ values. Then, after applying the binarization process, the distance $\Delta^{p}(A, G)$ has been computed.

Table 2 shows the results for the image in Fig. 1 , where $p=2, c=5$.

Table 2. Results obtained after the segmentation process.

\begin{tabular}{ll}
\hline$M G T$ value & Distance $\Delta^{p}(A, G)$ \\
\hline Gradient mean & 0.6038 \\
$M G T=0.95 * \max \left(\rho_{B}(x, y)\right)$ & 0.2722 \\
$M G T=0.9 * \max \left(\rho_{B}(x, y)\right)$ & 0.1988 \\
$M G T=0.85 * \max \left(\rho_{B}(x, y)\right)$ & 0.3412 \\
$M G T=0.8 * \max \left(\rho_{B}(x, y)\right)$ & 0.3704 \\
\hline
\end{tabular}

As shown, the lowest distance is obtained when $M G T=0.9 * \max \left(\rho_{B}(x, y)\right)$. Fig. 4 compares the ideal segmentation and the $M G T$ segmentation with the 
lowest $\Delta^{p}(A, G)$. Intuitively, if we compare the previous results in Fig. 3, the selected $M G T$ value is quite similar to the ideal segmentation.

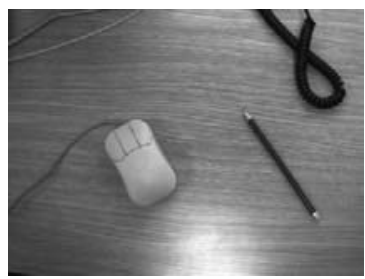

(a)

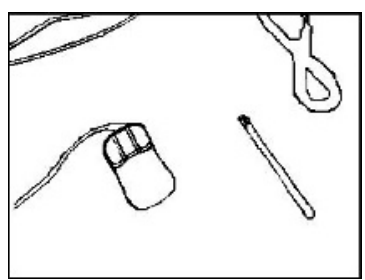

(b)

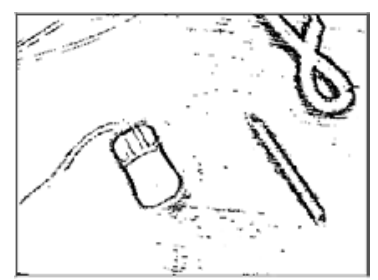

(c)

Fig. 4. (a) Original image. (b) Ideal segmentation. (c) MGT segmentation.

Let us consider now a more complex real image in order to confirm the accuracy of our technique to give an automated extraction of the threshold value with the best behavior. Fig. 5 and Table 3 show the results.

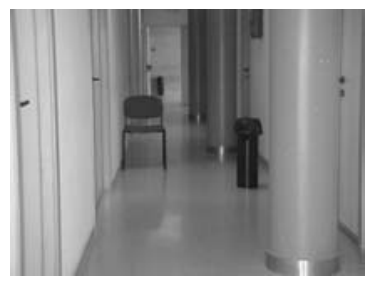

(a)

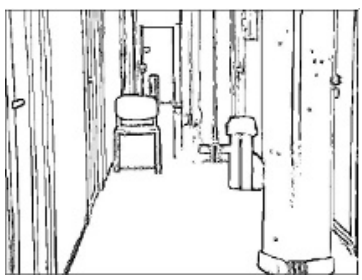

(b)

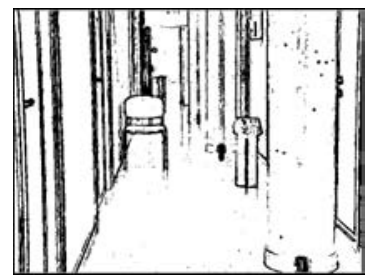

(c)

Fig. 5. (a) Original image. (b) Ideal segmentation. (c) MGT segmentation.

Table 3. Results obtained after the segmentation process.

\begin{tabular}{ll}
\hline$M G T$ value & Distance $\Delta^{p}(A, G)$ \\
\hline Gradient mean & 0.5526 \\
$M G T=0.95 * \max \left(\rho_{B}(x, y)\right)$ & 0.3115 \\
$M G T=0.9 * \max \left(\rho_{B}(x, y)\right)$ & 0.2245 \\
$M G T=0.85 * \max \left(\rho_{B}(x, y)\right)$ & 0.2731 \\
$M G T=0.8 * \max \left(\rho_{B}(x, y)\right)$ & 0.3219 \\
\hline
\end{tabular}

The minimum distance is obtained again when $M G T=0.9 * \max \left(\rho_{B}(x, y)\right)$ and, as a consequence, we can conclude that the parameters used for this segmentation are near optimal, as they have a behavior very close to ideal segmentation.

Nevertheless, the threshold could be adaptively updated so as to assume the real conditions in which every image has been taken by the vision system. 


\section{Conclusions}

In this paper, we have described a novel approach to image segmentation based on the selection of a suitable morphological gradient threshold. To do this, global morphological operators have been used to compute the gradient and the Laplacian and, after a proper binarization, the distance between the ideal segmentation and the MGT segmentation has been computed. As a consequence, the gradient threshold with the lowest distance has been selected as the optimal threshold value. Experimental results show that our model is fast and robust and could be applied for real-time imaging.

As a future work, we propose to extend the results of our research to object classification and recognition. It would be so desirable to consider new simulation experiments with different environments, such as image sequences obtained from a camera placed in a robot platform, where real-time constraints have a great influence in the final recognition results.

\section{References}

1. Pal, N.R., Pal, S.K.: A review on image segmentation techniques. Pattern Recognition, 9 (1993) 1277-1294

2. Arques, P., Compañ, P., Molina, R., Pujol, M., Rizo, R.: A cybernetic approach to the multiscale minimization of energy function: Grey level image segmentation. Kybernetes, 31 (2002) 596-608

3. Arques, P., Compañ, P., Molina, R., Pujol, M., Rizo, R.: Minimization of an energy function with robust features for image segmentation. Kybernetes, 32 (2003) 14811491

4. Ouadfel, S., Batouche, M.: MRF-based image segmentation using ant colony system. Electronic Letters on Computer Vision and Image Analysis 2 (2003) 12-24

5. Basu, M., Su, M.: Image smoothing with exponential functions. International Journal of Pattern Recognition and Artificial Intelligence, 15 (2001) 735-752

6. Pujol, F., García, J. M., Fuster, A., Pujol, M., Rizo, R.: Use of mathematical morphology in real-time path planning. Kybernetes, 31 (2002) 115-123

7. Pujol, F., Pujol, M., Llorens, F., Rizo, R., García, J. M. : Selection of a suitable measurement to obtain a quality segmented image. In Proc. of the $5^{\text {th }}$ Iberoamerican Symposium on Pattern Recognition, Lisbon, Portugal, (2000) 643-654 